\title{
OPTIONS OF REMOTE INTERACTION IN TYPICAL 1C CONFIGURATIONS
}

\author{
D.V. Kornienko $\mid$ Bunin Yelets State University \\ Candidate of physical and mathematical \\ Sciences \\ dmkornienko@mail.ru \\ Yelets
}

\begin{abstract}
The article is devoted to the description of the possibilities of increasing the efficiency of distance selling using the typical functionality of the programs "1C: Trade Management 11", "1C: Integrated Automation 2" or "1C: ERP Enterprise" and 1C services.
\end{abstract}

Keywords: remote interaction, integration, online service, customer orders.

\section{References}

1. 1C: Business network [1S: Biznes-set']. URL: https://1cbn.ru/ (date of access: 07.07.2020).

2. Information system 1C: ITS [Informatsionnaya sistema 1S:ITS]. URL: https://its.1c.ru/ (date of access: 10.08.2020).

3. Management system for sites UMI.CMS [Sistema upravleniya saytami UMI.CMS]. URL: https://umi.ru/ (date of access: 20.07.2020).

4. Online payments for business [Onlayn-platezhi dlya biznesa]. URL: https://kassa.yandex.ru/ (date of access: 27.08.2020).

DOI: $10.24888 / 2500-1957-2020-3-109-116$

УДК

339.17

\section{ЦЕНООБРАЗОВАНИЕ И ЦЕНОВАЯ ПОЛИТИКА В} ОРГАНИЗАЦИИ

Светлана Викторовна Мишина

старший преподаватель кафедры экономики и управления им. Н.Г. Нечаева svmishina2017@mail.ru г. Елец
Елецкий государственный университет им. И.А. Бунина

\begin{abstract}
Аннотация. Статья посвящена описанию методов ценообразования в типовой конфигурации «1C:ERP Управление предприятием». Подсистема ценообразования призвана решать на предприятии ряд важных задач: объединение товаров в ценовые группы (например, по-разному может формироваться цена на товары собственного производства и покупные товары); ведение классификатора различных видов цен (назначение различных вариантов расчета цен для товаров различных ценовых групп; настройка диапазонов конечных цен - установка ограничений по максимальным и минимальным ценам продажи и закупки; ведение классификатора скидок (наценок) (Различные условия предоставления скидок (наценок); Регистрация скидок (наценок); Совместное действие различных скидок (наценок); назначение автоматических скидок (наценок) при продаже оптом и в розницу).
\end{abstract}


Ключевые слова: ценообразование, виды цен, установка цен номенклатуры, прайс-лист.

Трудно переоценить значение, которое имеют цены в условиях рыночной экономики, они, в частности:

- определяют структуру производства;

- воздействуют на движение материальных потоков;

- влияют на распределение товарной массы;

- создают объемы производства, прибыли, рентабельности и т.п.

Грамотная ценовая политика, ценовая стратегия и тактика - основа успешной деятельности любого предприятия, тем более если предприятие выходит на внешний рынок, который не прощает даже малейших ошибок и просчетов.

Проблемы ценообразования весьма многообразны и интересны. Формирование цены весьма многоступенчатый, сложный процесс и в высокой степени определяется внешними по отношению к организации факторами. Учитывая влияние данных факторов, продавцу необходимо разработать ценовую политику.

Статья посвящена описанию вопросов, связанных с ценовой политикой, применяемой в организации. В понятие ценовой политики входит назначение оптимальных отпускных цен, установка гибких скидок и наценок.

Процесс ценообразования будет рассмотрен на примере типовой конфигурации «1C:ERP Управление предприятием».

Для каждой позиции номенклатуры может быть установлено несколько цен, предназначенных для использования в разных ситуациях. В прикладном решении «1C:ERP Управление предприятием» отдельно ведутся цены поставщиков (конкурентов) и отпускные цены организации.

При расчете отпускных цен можно использовать данные о зарегистрированных ценах поставщиков и конкурентов.

Независимо от того, о каких ценах идет речь - ценах закупки, продажи, ценах конкурентов, - понятие цены связывается с объектом номенклатуры и характеристикой номенклатуры без детализации по сериям. Считается, что объекты одной номенклатуры с разными сериями стоят одинаково.

При определении цены указание характеристики является обязательным, если для товара ведется учет в разрезе характеристик. Предусмотрена возможность группового указания одинаковой цены для одного товара с различными характеристиками.

Цены предприятия регистрируются документом «Установка цен номенклатуры». месте «Цены (прайс-лист)».

Предварительно все возможные варианты цены предприятия вводятся в классификатор «Виды цен». Для каждого вида цены определяются валюта цены, правила расчета, метод округления и т. д.

При оформлении документов продажи для каждого клиента автоматически заполняются те цены, которые определены для него в условиях продаж (типовом или индивидуальном соглашении). В соглашении можно задать как виды цен, которые ранее уже зарегистрированы документом «Установка цен номенклатуры», так и произвольные индивидуальные значения цен для конкретных товаров. При составлении условий продаж можно назначать различные виды цен для различных ценовых групп товаров.

При оформлении продаж клиентам (как оптом, так и в розницу) цены используются как начальные значения. Менеджер, оформляющий продажу оптом, или кассир, который оформляет продажи в розницу, может назначать различные скидки - как автоматические (в том числе и по карте лояльности), так и ручные. При оформлении продажи контролируется диапазон допустимых цен (минимальные и максимальные цены продажи), а также допустимый процент предоставления ручных скидок. 
Все номенклатурные позиции разделяются по ценовым группам. К одной ценовой группе следует отнести товары, которые используют одинаковые правила назначения цен. Например, на предприятии, которое занимается производством и торговлей мебелью, такими группами могут быть: мебель собственного производства, зарубежная мебель, эксклюзивная зарубежная мебель и т. п.

Правила формирования, расчета и использования той или иной цены хранятся в иерархическом классификаторе «Виды цен». В этом классификаторе регистрируются все типы цен - как цены продажи (колонки прайс-листа), так и вспомогательные цены (внутренние цены перепродажи между организациями предприятия, вспомогательные цены для расчета отпускных цен и т. д.). При задании правил расчета цен можно использовать данные, зарегистрированные в информационной базе. Например, использовать для расчета данные о ценах поставщиков, конкурентов или назначать цены только на те товары, которые включены в ассортимент магазина определенного формата.

Во избежание задания цен выше или ниже определенных значений в форме Виды цен настраивается диапазон допустимых цен по кнопке Настроить диапазон допустимых цен.

Для использования в документах доступны только действующие цены (статус Действует/Не действует в карточке Виды цен). В форме «Виды цен» список может быть настроен по статусу (Все/Действует/Не действует).

Назначение и согласование новых цен, а также актуализация текущих цен всегда подтверждаются документально. Для этих целей используется документ «Установка цен номенклатуры». Документ создается автоматически в рабочем месте «Цены (прайс-лист)». Расчет значений цен производится в соответствии с теми правилами, которые заданы для вида цен. При формировании цен можно фиксировать одновременно значения нескольких видов цен и применять правила по расчету одной цены на основании другой.

В соответствии с зарегистрированными ценами формируется печатная форма прайслиста. Зарегистрированные цены используются при оформлении продаж клиентам.

Список ценовых групп заполняется в разделе CRM и маркетинг - Настройки и справочники. Использование ценовых групп определяется функциональной опцией НСИ и администрирование - Настройка НСИ и разделов - CRM и маркетинг - Маркетинг Ценовые группы. Связь позиции номенклатуры с ценовой группой устанавливается в карточке номенклатуры. Одна номенклатурная позиция может быть отнесена только к одной ценовой группе. Предусмотрена возможность назначения ценовой группы нескольким товарам с помощью групповой обработки в списке Номенклатура (команда контекстного меню Изменить выделенные).

Создание новых и актуализация использующихся цен выполняются в рабочем месте CRM и маркетинг - Цены и скидки - Цены (прайс-лист).

Для заполнения списка товаров, по которым нужно назначить цены, используется кнопка «Сформировать».

В появившейся форме «Настройка параметров прайс-листа» на закладке «Отбор номенклатуры» необходимо установить параметры отбора номенклатуры. Если параметры не установлены, то будет заполнен весь список номенклатуры. Используя вариант настройки «Расширенный вид», можно устанавливать более сложные варианты отбора с группировкой условий («и», «или», «максимум», «минимум»), возможностью отбора по любым реквизитам номенклатуры и т. д. Установленные настройки отбора сохраняются на следующий сеанс работы. назначить.

На закладке «Колонки» прайс-листа настраивается список видов цен, которые нужно

В списке показаны зависимые и влияющие цены. Влияющие цены информируют о зависимости от них текущей цены, а зависимые должны пересчитываться после ее изменения. 
После выбора нужного вида цены можно отметить список цен, которые оказывают влияние на расчет данной цены и которые должны быть пересчитаны после задания новых цен данного вида. Для этих целей используются кнопки «Выбрать все влияющие» и «Выбрать все зависимые».

Если для вида цены в качестве способа расчета установлен «Произвольный запрос по данным ИБ» или «Наценка на цену поступления», то в колонке «Параметры» может потребоваться задать параметры СКД (схема компоновки данных). Если параметры не заданы, то перейти в форму настройки СКД для указания параметров можно с помощью команды табличной части «Редактировать настройки».

При этом цены, которые зависимы от данной цены, будут автоматически пересчитаны. После пересчета зависимые цены автоматически округляются в соответствии с правилами округления, которые заданы для вида цены.

При формировании прайс-листа для позиции номенклатуры заполняется значение текущей зарегистрированной цены и происходит автоматический пересчет всех цен, которые рассчитываются на основании исходных цен в соответствии с заданными правилами расчета. Для каждой позиции номенклатуры в сформированном списке можно задать цены вручную. При этом будут автоматически пересчитаны все зависимые цены.

Цены указываются в валюте, заданной для вида цен. Цена может быть назначена в разрезе упаковок товаров. Однако имеется ограничение: один вид цены может быть задан только для одного вида упаковки. Например, розничная цена фиксируется для штуки, а оптовая - для коробки. Информация об упаковках, для которых задана цена, запоминается, и при последующей установке цен автоматически предлагается установить цену за эту же упаковку. При оформлении документов цена пересчитывается в соответствии с коэффициентом, заданным для упаковки товара.

Если для товара ведется учет по характеристикам, то при задании цены на товар автоматически будут сформированы цены для всех характеристик.

При необходимости значение цены в табличной части формы «Цены (прайс-лист)» можно изменить. Сравнение измененной цены с ценой, действующей на определенную дату, отображается в табличной части формы и в информационной строке в нижней части формы. В информационной строке отражаются основные параметры формирования цены.

По гиперссылке «Настроить» в нижней части формы «Цены (прайс-лист)» настраивается отображение в списке колонок с ценами, действовавшими на определенную дату, а также сравнение их с измененными ценами.

Для регистрации новых цен следует выполнить команду «Применить измененные цены». При выполнении этой команды будет автоматически создан документ «Установка цен номенклатуры», в котором будет указан список зарегистрированных цен.

По гиперссылке «История изменения цен» можно посмотреть список ранее зарегистрированных документов «Установка цен номенклатуры». Из этого списка можно также создать новый документ «Установка цен номенклатуры». В течение дня можно устанавливать различные цены. Однако при оформлении документов всегда будет использоваться последняя назначенная цена - независимо от того, в какое время суток оформлен документ.

По гиперссылке «Прайс-лист» можно сформировать прайс-лист по отобранным в списке позициям.

Предусмотрена также возможность регистрации цен для конкретного товара из справочника «Номенклатура». При вводе нового товара можно сразу назначить для него цены. Для этих целей используется гиперссылка «Прайс-лист компании» в разделе «Цены» карточки номенклатуры.

Будет открыто рабочее место по установке цен с отбором по данной позиции номенклатуры. Работа по формированию новых цен производится аналогично предыдущему варианту. 
По кнопке «Применить измененные цены» будут автоматически зарегистрированы цены на новый товар.

Правом регистрации цен обладает только сотрудник (маркетолог), который отвечает за ценовую политику предприятия. Для этого пользователя должно быть установлено разрешенное действие (право) «Установка цен номенклатуры без согласования». Новый документ для этого пользователя создается со статусом «Согласован».

Для всех остальных пользователей документ создается в статусе «Не согласован». Для ввода цен в действие необходимо запустить бизнес-процесс согласования цен. При создании документа «Установка цен номенклатуры» из рабочего места «Цены (прайс-лист)» после записи документа бизнес-процесс согласования будет запущен автоматически.

Предусмотрена возможность изменять значения действующих цен.

По кнопке «Изменить цены - Загрузить действующие цены (задаваемые вручную)...» проводится изменение по ценам, установленным ранее вручную. Необходимо задать параметры расчета текущих цен: дату действия, процент изменения, вид цены, ограничения действия изменения по строкам и ячейкам табличной формы, округление.

По кнопке «Изменить цены - Загрузить из документов установки цен...» текущие цены устанавливаются по документам, по которым цены были установлены ранее. Необходимо из предложенного списка выбрать документы установки цен.

По кнопке «Изменить цены - Рассчитать вычисляемые по данным ИБ и формулам...» производится расчет текущих цен, в которых установлены виды цен с вариантом задания цены «Произвольный запрос к данным ИБ», «Наценка на цену поступления», «Наценка на другой вид», «Произвольная формула от других видов цен».

По кнопке «Изменить цены - Округлить цены (задаваемые вручную)...» производится округление цен, назначаемых вручную. Необходимо выбрать из списка виды цен, по которым в качестве способа задания цены установлен «Ручное назначение». Округление осуществляется по правилам, которые указаны для вида цены.

По кнопке «Изменить цены - Изменить на процент...» можно увеличивать или уменьшать цены на фиксированный процент. Для каждого вида цены можно указать свой процент, на который следует изменить действующие цены. Рассчитанные измененные цены можно округлить. Округление происходит в соответствии с теми правилами округления, которые указаны для вида цены.

По кнопке «Изменить цены - Изменить цены» можно назначить новые цены только для выбранных видов цен по выделенным строкам списка.

Действующие цены можно изменить вручную в ячейке.

Если в форме «Цены (прайс-лист)» установлен флаг «Рассчитывать цены автоматически» (пункт меню Параметры), то в соответствии с изменениями цен будут автоматически пересчитаны все зависимые цены.

В том случае, если на предприятии используются сложные формулы расчета, для изменения цен можно использовать таблицы Excel. Предусмотрена возможность выгружать данные в таблицы и загружать измененные данные в обработку «Цены (прайс-лист)» или документ «Установка цен номенклатуры». Для выгрузки данных в таблицу Excel используется команда «Excel - Выгрузить в Excel». Информация об измененных ценах, которые будут загружены, регистрируется в таблицах Excel в поле «Цена». Для загрузки измененных цен используется команда «Excel - Загрузить из Excel». Информация об измененных ценах будет загружена в колонку «Цена».

Расчет цен продажи может также производиться в соответствии с ценами поставки и заданными наценками. При этом возможны следующие варианты расчета цен:

- расчет цен продажи на основании конкретного документа поставки;

- расчет цен продажи на основании обобщенных данных о последних поставках товаров от разных поставщиков; 
- расчет цен продажи при оформлении внутренних документов (порча товаров, сборка товаров и т. д.).

При вводе документа «Установка цен номенклатуры» на основании документа поставки автоматически рассчитываются отпускные цены на основании той цены, которая зарегистрирована в документе поставки.

При регистрации цен учитываются значения флагов «Цены включают НДС» в виде цены и в документе:

- если значения флажков совпадают, то значение цены фиксируется так, как оно указано в документе;

- если в виде цен указано Цены включают НДС, а в документе поставки цены указываются без учета НДС, то значение цены поставки увеличивается на сумму НДС.

В список цен добавляются все те виды цен, у которых установлен флаг «При вводе на основании документов поставки».

На основании цены поставки автоматически рассчитываются все те цены, для которых указан способ задания цены «Наценка на цену поступления», а также те цены, в формулах расчета которых указана цена поступления (рассчитываемая по данным информационной базы на основании предопределенной СКД).

При этом автоматически пересчитываются все цены, которые являются зависимыми от рассчитанных цен. Например, если розничная цена рассчитывается на основании цены поступления, а дистрибьюторская цена рассчитывается на основании розничной цены, то при регистрации цены поступления будут пересчитаны отпускные цены: розничная и дистрибьюторская.

В документе «Установка цен номенклатуры» дополнительно показываются отклонения вновь регистрируемых цен (Цена) от ранее зарегистрированных (Цена на дату) и процент отклонения новой цены от старой (\%). Если новая цена уменьшается по отношению к старой, то процент отклонения показывается с минусом и помечается красным цветом.

Предусмотрена также возможность настройки порога срабатывания для каждого вида цены. Можно указать, при каком именно проценте отклонения новой цены по отношению к старой должны изменяться цены. Информация о порогах срабатывания может быть детализирована для каждой ценовой группы товаров.

Например, если для цены поступления установить порог срабатывания для товаров ценовой группы «Вентиляторы» равным 3 \%, то это означает, что новые цены на товары этой ценовой группы будут изменены только в том случае, если они отличаются от старых не менее чем на $3 \%$. Если процент изменения меньше $3 \%$, то при формировании документа Установка цен номенклатуры значения новых цен будут установлены равными значениям старых. Строки с товарами, по которым цены не изменились, можно удалить из документа, используя команду «Изменить строки - Удалить строки без изменения».

При проведении документа «Установка цен номенклатуры» будут зарегистрированы все цены, указанные в документе. Аналогичным образом можно рассчитать цены на основании тех цен, которые зарегистрированы в документе «Заказ поставщику».

Предусмотрена возможность расчета цен на основании данных о последней цене поставки различных поставщиков. В этом случае в новом документе или в обработке «Цены (прайс-лист)» фиксируется список позиций, по которым нужно зарегистрировать цену поставки и рассчитать отпускные цены. В список видов цен следует добавить вид цен «Цена поступления».

Для товаров автоматически заполняются цены, по которым была зарегистрирована последняя поставка. Заполняются цены, которые зарегистрированы в последнем проведенном документе Приобретение товаров и услуг. Автоматически пересчитываются все остальные зависимые цены в соответствии с заданными формулами расчета. 
Документ «Установка цен номенклатуры» может быть введен на основании следующих внутренних документов товародвижения:

- Порча товаров - для расчета цен продажи некачественных товаров. Данные о цене (себестоимости) испорченных товаров регистрируются в качестве цены поступления;

- Сборка (разборка) товаров - для расчета цены комплекта (или комплектующих) на основании данных о себестоимости комплекта (комплектующих);

- Прочее оприходование товаров - для расчета цен продажи товаров, зарегистрированных при прочих поступлениях (без указания поставщика).

Процесс согласования цен рекомендуется использовать в том случае, если на предприятии предварительный расчет цен делают маркетологи, a окончательное утверждение производится руководящим составом предприятия (финансовым директором, руководителем предприятия).

Использование процесса согласования определяется функциональной опцией НСИ и администрирование - Настройка НСИ и разделов - CRM и маркетинг - Согласование цен.

Если процесс согласования не используется, то цены, зафиксированные в документе «Установка цен номенклатуры», начинают действовать сразу после проведения документа. Для документа автоматически устанавливается статус «Согласован» (информация о статусе в документе не отображается).

Если процесс согласования используется, то документ может быть проведен в двух статусах: «Не согласован» или «Согласован». При проведении документа отображается информация о его текущем состоянии: «Ожидается согласование» или «Действует».

Для согласования новых и измененных цен используется бизнес-процесс «Согласование цен». При назначении цен с помощью обработки «Цены (прайс-лист)» бизнес-процесс согласования цен запускается автоматически. Если для установки цен используется документ «Установка цен номенклатуры», то бизнес-процесс согласования запускается путем ввода на основании (Создать на основании - Согласование цен).

Информация о том, кто будет принимать решение об утверждении цен, вводится в список «Исполнители» роли «Согласующий установки цен номенклатуры» (раздел НСИ и администрирование - Настройка НСИ и разделов - CRM и маркетинг - Ответственные за согласование цен).

В документе «Установка цен номенклатуры» и в рабочем месте «Цены (прайс-лист)» можно сформировать отчеты для анализа установленных цен.

Для того чтобы сформировать нужный отчет, необходимо в табличной части документа или обработки выделить строки с номенклатурой, по которой нужно сформировать отчет, и далее воспользоваться соответствующей командой контекстного меню.

Отчет по динамике цен в наглядном виде демонстрирует изменение цен номенклатуры во времени. Для каждого вида цен выводится своя диаграмма.

Отчет «История изменения цен» показывает график изменения цен: даты изменения и новые значения цен.

Используя команду «Товары со схожими свойствами», можно отобрать в списке аналоги товара, выделить их в списке и проанализировать цены по всем аналогичным товарам. Список параметров, которые используются для отбора аналогов (товаров со схожими свойствами), определяется для каждого вида номенклатуры на закладке «Фильтр по свойствам».

\section{Список литературы}

1. Баздникин А. С. Цены и ценообразование. М.: Юрайт, 2017. 332 с.

2. Информационная система 1C: ИTC. URL: https:/its.1c.ru/ (дата обращения: 10.08.2020). 


\title{
PRICING AND PRICING POLICY IN THE ORGANIZATION
}

\author{
S.V. Mishina $\mid$ Bunin Yelets State University \\ Senior Lecturer at the Department of \\ Economics and Management named after \\ N.G. Nechaeva \\ svmishina2017o@mail.ru \\ Yelets
}

\begin{abstract}
The article is devoted to the description of pricing methods in a typical configuration "1C: ERP Enterprise Management". The pricing subsystem is designed to solve a number of important tasks at the enterprise: combining goods into price groups (for example, the price of goods of own production and purchased goods can be formed in different ways); maintaining a classifier of various types of prices (assigning different options for calculating prices for goods of different price groups; setting up ranges of final prices - setting restrictions on the maximum and minimum prices for sale and purchase; maintaining a classifier of discounts (markups) (Various conditions for granting discounts (markups); Registration of discounts (margins); Joint action of various discounts (margins); assignment of automatic discounts (margins) for wholesale and retail sales).
\end{abstract}

Keywords: pricing, types of prices, setting item prices, price list.

\section{References}

1. Bazdnikin, A. S. (2017). Prices and pricing [Tseny i tsenoobrazovaniye]. Moscow.

2. Information system 1C: ITS [Informatsionnaya sistema 1S: ITS.]. URL: https://its.1c.ru/ (date of access: 10.08.2020).

DOI: $10.24888 / 2500-1957-2020-3-116-123$

\begin{tabular}{l|l} 
УДК & ОСНОВНЫЕ ФУНКЦИОНАЛЬНЫЕ ВОЗМОЖНОСТИ СДО \\
371.39 & МООDЦЕ ПРИ РАЗРАБОТКЕ ЭЛЕКТРОННЫХ УЧЕБНЫХ \\
& КУРСОВ ДЛЯ ИСПОЛЬЗОВАНИЯ В ОБРАЗОВАТЕЛЬНОЙ \\
& СРЕДЕ
\end{tabular}

Татьяна Васильевна Рихтер $\mid$ Пермский государственный национальный к.П.н., доцент исследовательский университет tatyanarikhter@mail.ru

$$
\text { г. Пермь }
$$

\begin{abstract}
Аннотация. В статье рассматриваются основные функциональные возможности CДо Moodle при разработке электронных учебных курсов для использования в образовательной среде: управление сайтом (осуществление администратором, настройка цветов, шрифтов, расположения объекта на странице сайта); управление пользователями (регистрация пользователей: само-регистрация, ручная регистрация администратором, автоматическое напоминание пароля пользователю, защита от несанкционированного доступа); управление курсами
\end{abstract}

